\title{
Ojo seco y cirugía refractiva, estudio de red de citación
}

\section{Dry eye and refractive surgery, a citation network study}

\author{
Elena Fraga-Graells*, Cristina Álvarez-Peregrina, César Villa-Collar y Miguel A. Sánchez-Tena \\ Facultad de Ciencias Biomédicas y de la Salud, Universidad Europea de Madrid, Madrid, España
}

\begin{abstract}
Resumen
Objetivo: Identificar las conexiones entre los investigadores más citados y el impacto de autores, revistas y contenidos en el campo de ojo seco tras cirugía refractiva, para focalizar la futura investigación en ello. Método: Se realizó una búsqueda de "ojo seco y cirugía refractiva" en Web of Science, y se limitó el campo para artículo, palabras clave y resumen, vinculados con "OR". El archivo resultante se analizó con el software CitNetExplorer ${ }^{\oplus}$ y se obtuvieron las publicaciones más citadas. Se realizó otro subanálisis sobre citaciones entre autores para identificar grupos y publicaciones principales. Resultados: Se hallaron 220 publicaciones entre 1997 y 2019 que generaron 675 citaciones en la red. Los 20 artículos más citados, encabezados por Hovanesain, et al., fueron publicados por Journal of Cataract and Refractive Surgery y Journal of Refractive Surgery. Mediante las funciones "cluster" y "core" se obtuvieron dos grupos y 106 artículos con al menos cuatro citas en la red, respectivamente. Conclusiones: Las técnicas actuales de cirugía refractiva son procedimientos avanzados y su complicación más frecuente es la aparición de ojo seco, que provoca insatisfacción y disminución de la calidad de vida de los pacientes. El ojo seco y la cirugía refractiva son un área destacada para los investigadores, con una cantidad significativa de publicaciones y conexiones entre ellos. Ampliar el conocimiento en este ámbito servirá para la mejora de aplicaciones clínicas con el fin de disminuir la aparición de ojo seco.
\end{abstract}

Palabras clave: Ojo seco. Cirugía refractiva. LASIK. Red de citación.

\section{Abstract}

Purpose: To identify the connections between the most cited researchers, and the impact of authors, journals, and subjects in the field of dry eye after refractive surgery, to focus future research on it. Method: A search of "dry eye and refractive surgery" was made through Web of Science, and limiting the field for the article, keywords and abstract, linked with the OR tab. The file obtained was analyzed later with CitNetExplorer ${ }^{\circledR}$ software obtaining an analysis of top-cited publications. A subnetwork analysis was made depending on the citations among them to identify clústeres and core publications. Results: This study showed 220 references in the period 1997 to 2019 that generated 675 citations across the network. The top 20 citations score has been published by the Journal of Cataract and Refractive Surgery and Journal of Refractive Surgery. Hovanesian, et al. tops the ranking of top-cited articles on dry eye and refractive surgery. Clustering and Core functions gave 2 clusters and 106 papers with at least 4 citations in the network, respectively. Conclusions: Current refractive surgery techniques are developed procedures, but often post-surgical complication is dry eye, that causes dissatisfaction and detriment to the patient's quality of life. Dry eye and refractive surgery are remarkable fields for researchers showing a prominent quantity of publications and connections among those. Expand knowledge in this field will serve to improve clinical applicability to decrease dry eye.

Keywords: Dry eye. Refractive surgery. LASIK. Citation network.

Correspondencia:

*Elena Fraga-Graells

Tajo $\mathrm{s} / \mathrm{n}$

Fecha de recepción: 19-06-2020

Fecha de aceptación: 21-06-2021

DOI: 10.24875/RMO.M21000189
Disponible en internet: 07-09-2021 Rev Mex Oftalmol. 2021;95(6):235-244

www.rmo.com.mx

E-mail: efraga @ serviciosoptometria.com

0187-4519/( 2021 Sociedad Mexicana de Oftalmología. Publicado por Permanyer. Este es un artículo open access bajo la licencia CC BY-NC-ND (http://creativecommons.org/licenses/by-nc-nd/4.0/). 


\section{Introducción}

Según la Tear Film \& Ocular Surface Society, «El ojo seco es una enfermedad multifactorial de la superficie ocular caracterizada por una pérdida de homeostasis de la película lagrimal, y se acompaña de síntomas oculares, en los que la inestabilidad y la hiperosmolaridad de la película lagrimal, la inflamación y el daño de la superficie ocular y las anomalías neurosensoriales desempeñan funciones etiológicas»'. Es un síndrome amplio y complejo, y puede manifestar síntomas de malestar ocular y alteraciones visuales desde su etapa más leve, incluso con posibles lesiones en la superficie ocular en los casos más graves. Su tratamiento puede requerir medidas ambientales, sustitutos de lágrimas o terapias farmacológicas.

Las técnicas actuales de cirugía refractiva, como el intercambio refractivo del cristalino, la queratectomía fotorrefractiva (PRK) y la queratomileusis in situ asistida con láser (LASIK), son procedimientos muy desarrollados que han logrado altos niveles de eficacia, seguridad y previsibilidad. Su complicación posquirúrgica más frecuente es el ojo seco, que aunque parece ocurrir de forma transitoria, puede volverse crónico y provocar insatisfacción y detrimento de la calidad de vida del paciente. Estas técnicas alteran la superficie ocular y la homeostasis de la película lagrimal. LASIK es el procedimiento más común de cirugía refractiva. Influye en el estado de la película lagrimal posoperatoria afectando aproximadamente al $50 \%$ de los pacientes en la primera semana tras la intervención, al $40 \%$ en el primer mes posoperatorio y al $20-40 \%$ a los 6 meses $^{2,3}$. La incidencia de síntomas crónicos de ojo seco 1 año después de LASIK es del $0.8 \%{ }^{4}$. Entre otras razones, la sensibilidad corneal, la glándula lagrimal y los circuitos del reflejo de parpadeo después de la cirugía pueden afectar la calidad y la cantidad de la película lagrimal. La búsqueda de nuevos métodos para mejorar la pérdida de efectos neurotróficos en la etapa posquirúrgica es actualmente el objetivo de varias investigaciones. Por tanto, para analizar los diagnósticos diferenciales y personalizar el tratamiento del paciente es fundamental una valoración exhaustiva y crítica de los resultados publicados.

Muchos investigadores de todo el mundo han estudiado el ojo seco después de la cirugía refractiva, pero se desconoce cómo están conectados estos investigadores y los resultados. Un análisis de la red de citación aporta información más simple y visual, y proporciona una mejor comprensión de la investigación realizada en un campo específico.

\section{Objetivo}

En este trabajo se analizan las principales conexiones entre los investigadores más citados y se obtiene una red de citación para evaluar el impacto de los diferentes autores, revistas y sujetos en el campo del ojo seco tras la cirugía refractiva. Este estudio de la red de citación simplifica la información y cuantifica los artículos más relevantes publicados. También sirve para crear grupos de acuerdo con sus conexiones, así como sus frecuencias de citas y clústeres de identificación de estudios. El objetivo principal es identificar todos los conceptos antes mencionados para enfocar futuras investigaciones.

\section{Método}

Estas investigaciones bibliométricas se definieron para agrupar publicaciones en función de sus relaciones de citación y analizar los resultados del clúster a nivel de publicaciones individuales.

La investigación bibliométrica se realizó a través de Web of Science (WOS), una plataforma basada en tecnología web que incluye referencias de las principales publicaciones científicas de cualquier disciplina del conocimiento desde 1945. Mediante técnicas de descarga remota se seleccionaron los artículos publicados entre 1997 y 2019. Se realizó una búsqueda en idioma inglés con dos palabras de texto libre, "ojo seco" como primer descriptor y "cirugía refractiva" como el secundario, ambas vinculadas con la pestaña OR booleana y limitando el campo para artículo, palabras clave y resumen. No se aplicaron otros límites de búsqueda. La fecha de la búsqueda fue el 29 de marzo de 2020. El archivo contiene publicaciones hasta 2019 inclusive. Esto se determina para que todos los resultados de todo el año aparezcan en el análisis de comparación.

Se descargó un archivo de WOS y los elementos duplicados se eliminaron manualmente. Se realizó una primera revisión comparando los nombres de los autores y su número de publicaciones por año. Se realizó una segunda revisión de verificación identificando publicaciones con igual número de citas y comparando a sus autores. Posteriormente, el archivo fue analizado mediante el software CitNetExplorer ${ }^{\circledR}$, que se utiliza para visualizar y analizar las publicaciones más importantes en un campo específico, revelando las relaciones que existen entre ellas ${ }^{5}$.

Para mostrar las citas en la red en un periodo, se realizó un análisis cuantitativo. Las conexiones internas entre autores, revistas y temas a través de las 


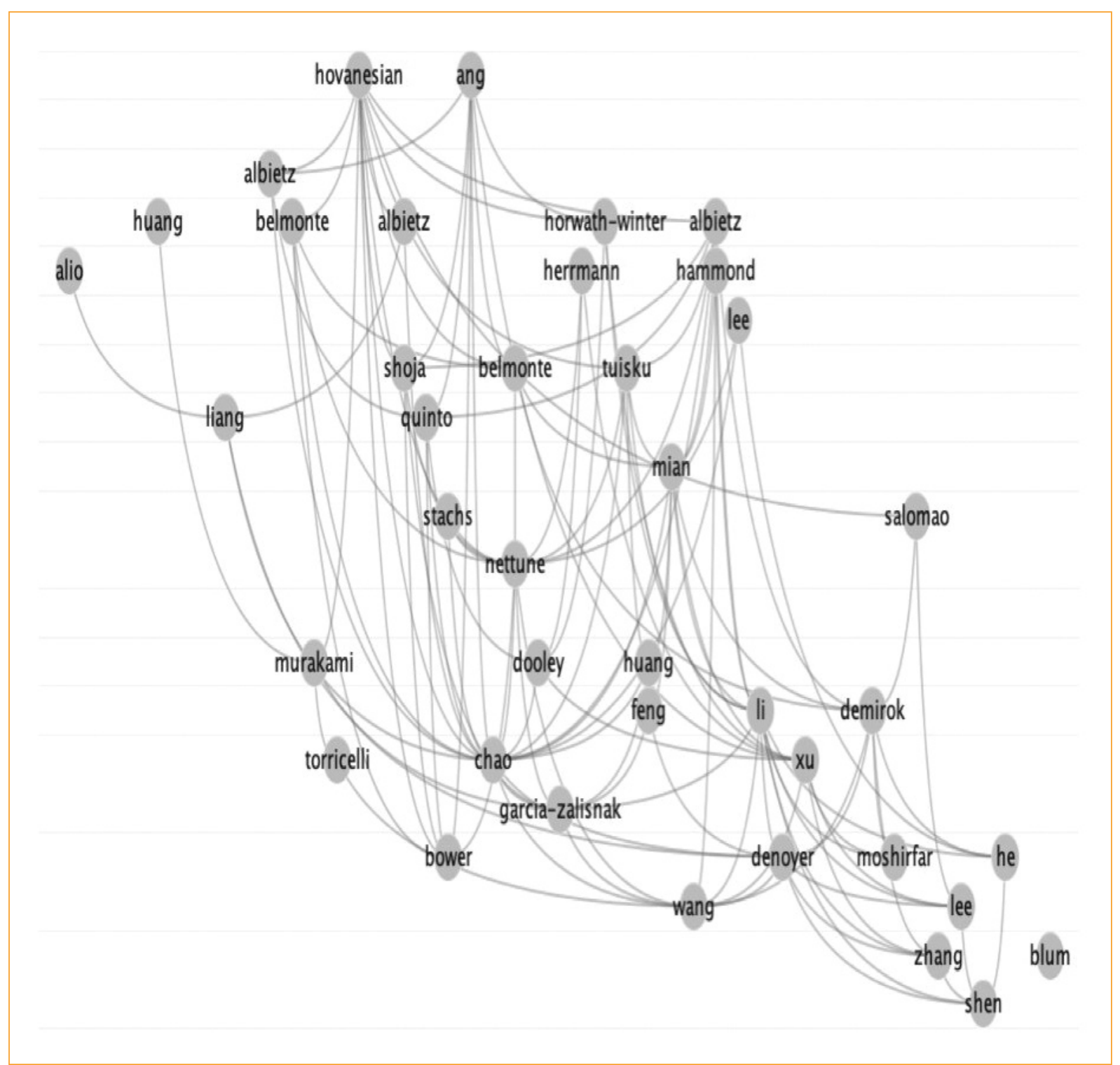

Figura 1. Las 20 publicaciones más citadas en la red de ojo seco y cirugía refractiva.

publicaciones dentro de la red se estudiaron por el atributo "puntaje de citas" de CitNetExplorer ${ }^{\circledR 6}$.

Se obtuvo un análisis de subred utilizando la función Cluster, que ofrece una lista de grupos o clústeres diferenciados según las asociaciones entre ellos?.

Finalmente, también se analizaron las publicaciones más importantes de la red utilizando la función Core. Esta función ofrece el porcentaje de publicaciones con cuatro o más citas dentro de la red.

\section{Resultados}

La red de ojo seco y cirugía refractiva cuenta con 220 referencias, lo que genera 675 citas en toda la red, siendo 644 de estas engendradas desde 2001 hasta el final de la búsqueda (lo que representa el $95 \%$ del total).

Respecto a los autores, Hovanesian, et al. ${ }^{8}$ en 2001 encabeza la clasificación del artículo más citado sobre ojo seco y cirugía refractiva, con una puntuación de 42 citas. El título de dicho artículo es "Síntomas de ojo seco y síndrome de erosión recurrente después de cirugía refractiva».

La figura 1 muestra la red.

La tabla 1 muestra que las 20 citas principales han sido publicadas principalmente en Journal of Cataract and Refractive Surgery y Journal of Refractive Surgery. 
Tabla 1. Las 20 publicaciones más citadas de 1997 a 2019 en la red de ojo seco y cirugía refractiva

\begin{tabular}{|c|c|c|c|}
\hline Autores & Revista & Año & Puntaje citas \\
\hline $\begin{array}{l}\text { Hovanesian, } \\
\text { et al. } .^{8}\end{array}$ & $\begin{array}{l}\text { Journal of Cataract } \\
\text { and Refractive } \\
\text { Surgery }\end{array}$ & 2001 & 42 \\
\hline Denoyer, et al..$^{9}$ & Ophthalmology & 2015 & 30 \\
\hline Li, et al. ${ }^{10}$ & Plos One & 2013 & 29 \\
\hline Ang, et al. ${ }^{11}$ & $\begin{array}{l}\text { Current Opinion in } \\
\text { Ophthalmology }\end{array}$ & 2001 & 26 \\
\hline $\mathrm{Xu}$, et al. ${ }^{12}$ & $\begin{array}{l}\text { Journal of Refractive } \\
\text { Surgery }\end{array}$ & 2014 & 23 \\
\hline Demirok, et al. ${ }^{13}$ & $\begin{array}{l}\text { Optometry and } \\
\text { Vision Science }\end{array}$ & 2013 & 22 \\
\hline Nettune, et al. ${ }^{14}$ & The Ocular Surface & 2010 & 20 \\
\hline Tuisku, et al. ${ }^{15}$ & $\begin{array}{l}\text { Journal of Refractive } \\
\text { Surgery }\end{array}$ & 2007 & 19 \\
\hline Albietz, et al. ${ }^{16}$ & $\begin{array}{l}\text { Journal of Cataract } \\
\text { and Refractive } \\
\text { Surgery }\end{array}$ & 2004 & 18 \\
\hline Mian, et al. ${ }^{17}$ & $\begin{array}{l}\text { Cataract and } \\
\text { Refractive Surgery }\end{array}$ & 2009 & 17 \\
\hline Chao, et al. ${ }^{18}$ & The Ocular Surface & 2014 & 16 \\
\hline Shoja, et al. ${ }^{3}$ & $\begin{array}{l}\text { European Journal of } \\
\text { Ophthalmology }\end{array}$ & 2007 & 15 \\
\hline Quinto, et al. ${ }^{19}$ & $\begin{array}{l}\text { Current Opinion in } \\
\text { Ophthalmology }\end{array}$ & 2008 & 14 \\
\hline Belmonte, et al. ${ }^{20}$ & $\begin{array}{l}\text { Experimental Eye } \\
\text { Research }\end{array}$ & 2004 & 13 \\
\hline Belmonte $^{21}$ & $\begin{array}{l}\text { Journal } \\
\text { of Refractive Surgery }\end{array}$ & 2007 & 12 \\
\hline Zhang, et al. ${ }^{22}$ & $\begin{array}{l}\text { Journal of Refractive } \\
\text { Surgery }\end{array}$ & 2016 & 12 \\
\hline Moshirfar, et al. ${ }^{23}$ & $\begin{array}{l}\text { Journal of Cataract } \\
\text { and Refractive } \\
\text { Surgery }\end{array}$ & 2015 & 11 \\
\hline Bower, et al. ${ }^{4}$ & $\begin{array}{l}\text { Journal of Cataract } \\
\text { and Refractive } \\
\text { Surgery }\end{array}$ & 2015 & 11 \\
\hline Lee, et al. ${ }^{24}$ & $\begin{array}{l}\text { American Journal of } \\
\text { Ophthalmology }\end{array}$ & 2006 & 10 \\
\hline Albietz, et al. ${ }^{25}$ & $\begin{array}{l}\text { Journal of Refractive } \\
\text { Surgery }\end{array}$ & 2004 & 9 \\
\hline
\end{tabular}

En cuanto a la puntuación de las citas de los 20 artículos más citados, se extiende de 42 a 9 . Solo tres de los más citados (15\%) no están por encima del promedio y no hacen referencia a sus artículos sobre LASIK:
Zhang, et al. ${ }^{22}$ con un metaanálisis de la técnica SMILE y FS-LASIK, Bower, et al. ${ }^{4}$ con un artículo de PRK, y Lee, et al. ${ }^{24}$ con un artículo de LASEK; su puntuación de citas es 12,11 y 10 , respectivamente.

Sobre las revistas en las que se han publicado los 20 artículos más citados, cinco de ellos corresponden a Journal of Refractive Surgery y otros cinco a Journal of Cataract and Refractive Surgery; les sigue Current Opinion in Ophthalmology, con dos publicaciones. Ninguna otra revista publicó más de un artículo.

\section{Clústeres}

La función Cluster proporciona dos clústeres:

- Clúster 1, con 138 publicaciones (62\% del total de la red). Contiene 558 citas ( $82 \%$ de la red total) de 2001 a 2017. Estas publicaciones tratan sobre la etiología del ojo seco inducido por LASIK. Los 20 más citados en la red coinciden en apariencia y orden con los 20 más citados en esta subred. Hovanesian, et al. encabeza este ranking con su publicación en Journal of Cataract and Refractive Surgery en $2001^{8}$. En el top 40 de este grupo, solo seis y cuatro publicaciones también se referían específicamente a sensación e inervación corneal, y cuatro, seis y tres se referían a las técnicas SMILE, láser de femtosegundo y PRK, respectivamente. Las revistas más habituales fueron Journal of Refractive Surgery con ocho artículos, Journal of Cataract and Refractive Surgery con siete y Current Opinion in Ophthalmology con tres. Los años más productivos fueron 2015 y 2014 con seis publicaciones por año, y 2004 y 2013 con cuatro publicaciones por año.

- Clúster 2, con 26 publicaciones (11\% del total de la red). Contiene 55 citas ( $8 \%$ de la red total) de 2005 a 2019. Estas publicaciones tratan sobre cirugía refractiva y otras afecciones relacionadas con el ojo seco, como el síndrome de Sjögren, alergias y enfermedades reumatológicas, u otros tratamientos posoperatorios, como la ciclosporina para el tratamiento del ojo seco. Liang, et al. ${ }^{26}$ encabezan el ranking con su publicación en Cornea, en 2008, y con una puntuación de citas de 9. Los años más productivos fueron 2019 con cinco publicaciones por año, seguido de 2014 con cuatro y 2016, 2013 y 2008 con tres publicaciones por año.

Las 56 publicaciones restantes no están asociadas entre sí, por lo que no pertenecen a ningún clúster.

Las figuras 2 y 3 muestran las conexiones de cada clúster. 


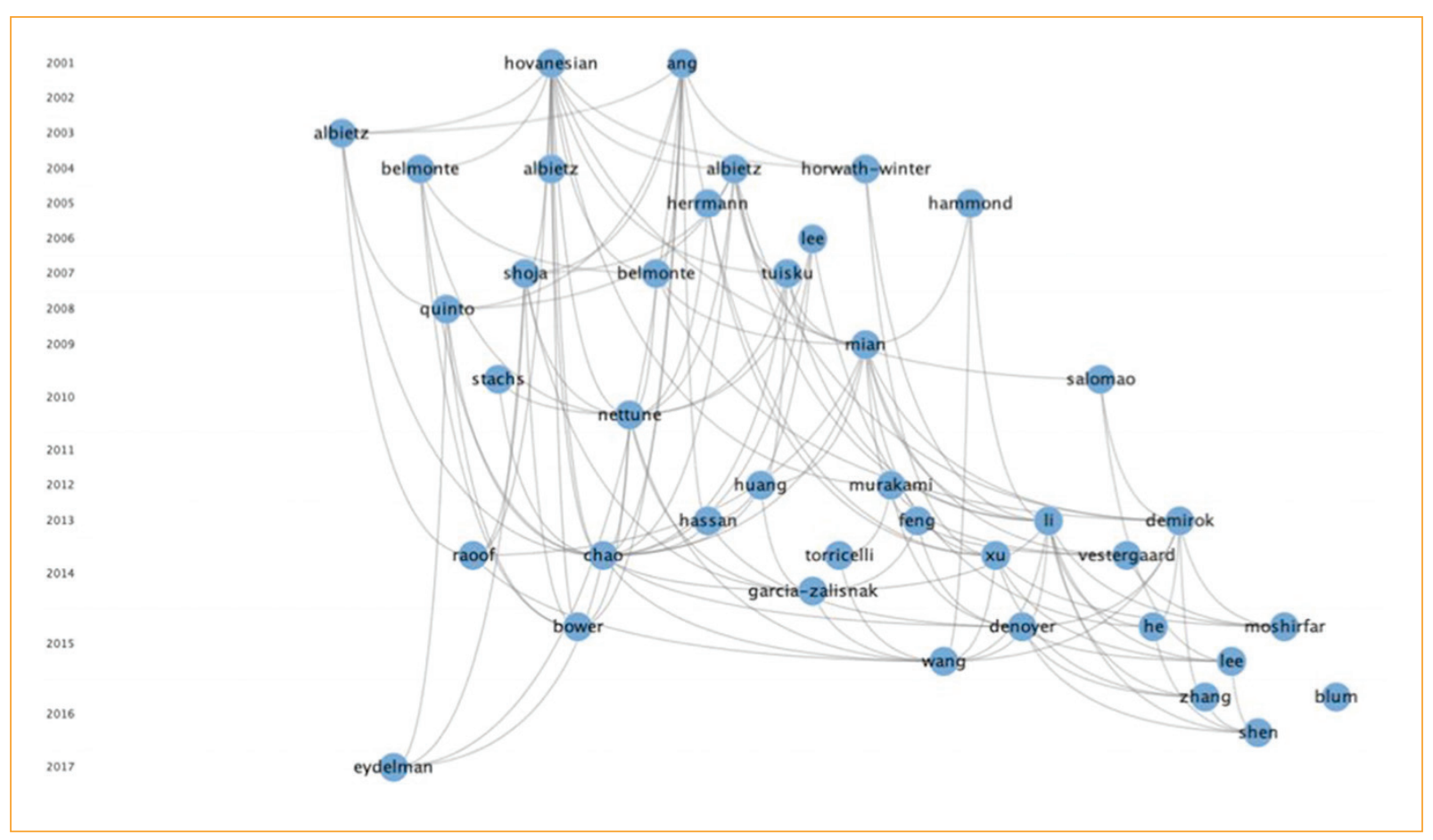

Figura 2. Los 40 artículos más citados de la subred del clúster 1.

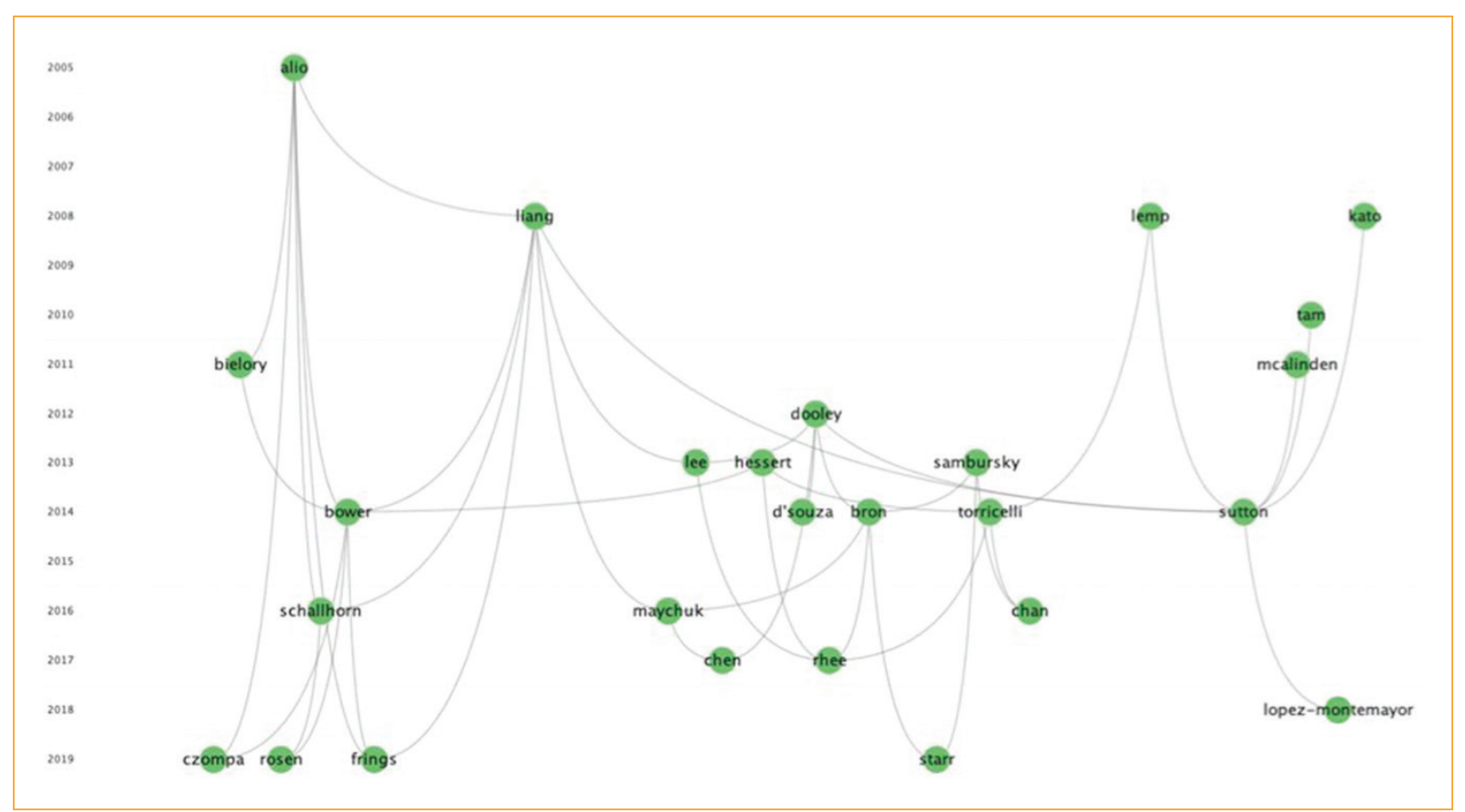

Figura 3. Subred del clúster 2.

Los años con más publicaciones en el top 40 de la subred del clúster 1 fueron 2014 y 2015, con seis por año, seguidos de 2004 con cuatro, y 2007, 2010 y 2013 con tres por año. La figura 4 muestra el número de publicaciones por año de los 26 artículos principales de cada grupo. 


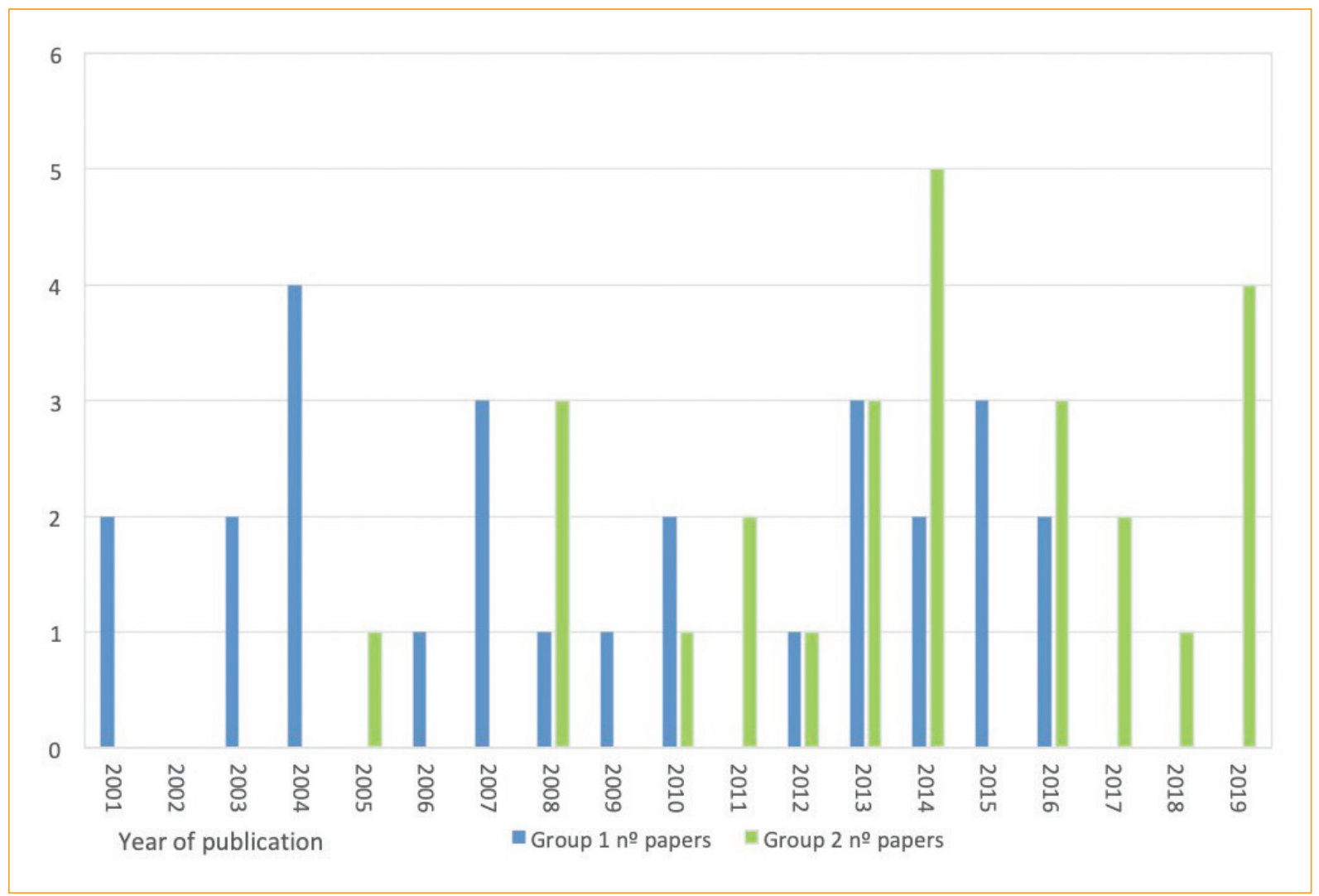

Figura 4. Número de publicaciones por año de los 26 artículos principales de cada clúster.

\section{Publicaciones Core}

La función Core muestra 106 artículos con al menos cuatro citas en la red, lo que representa un $47.92 \%$. Hovanesian, et al. del clúster 1 aparece en la primera posición, y Liang es el más citado del clúster 2, en la posición 21. Hay 44 publicaciones del clúster 1 (31.88\%) y 14 publicaciones del clúster 2 (53.84\%) que no aparecen en los resultados Core porque tienen menos de cuatro citas. La figura 5 muestra los resultados de la función Core.

\section{Discusión}

El ojo seco es una de las enfermedades oftálmicas más prevalentes que afecta la calidad de vida de los pacientes. También es una de las complicaciones más frecuentes tras la cirugía refractiva.

Este estudio proporciona información sobre la agrupación de publicaciones basadas en la red de citación. Permite analizar los resultados de las publicaciones más importantes sobre ojo seco tras la cirugía refractiva. La red se inicia en 1997, porque es el año de la primera publicación que hace referencia a "ojo seco" y "cirugía refractiva", y finaliza en 2019. En cuanto a la aparición de las técnicas PRK en 1980 y LASIK en 1990, se puede suponer que no hubo publicaciones sobre ojo seco y cirugía refractiva hasta que la incidencia de signos y síntomas de ojo seco se observó ampliamente en un número suficiente de casos de cirugía. No se han encontrado otras publicaciones antes de 1997, excepto una de 1995 firmada por Lemp ${ }^{27}$. El nombre del autor, la revista y el año de esa publicación aparecieron en la búsqueda de WOS, pero el título no estaba disponible, y se eliminó por no tener suficientes detalles. Esta circunstancia puede provocar un sesgo de resultados en el estudio realizado, que también puede repetirse en futuros estudios sobre el mismo tema.

Los 20 artículos más citados en la red se publicaron entre 2001 y 2016, reconociendo cinco etapas diferentes. Estas cinco etapas indican los diferentes temas estudiados y cómo ha evolucionado la investigación en el campo del ojo seco y la cirugía refractiva. La primera etapa corresponde al año 2001, con dos publicaciones: Hovanesian, et al. ${ }^{8}$ y Ang, et al. ${ }^{11}$ El artículo de Hovanesian, et al. ${ }^{8}$ estudió la incidencia y la gravedad del ojo seco en 1731 pacientes que habían sido 


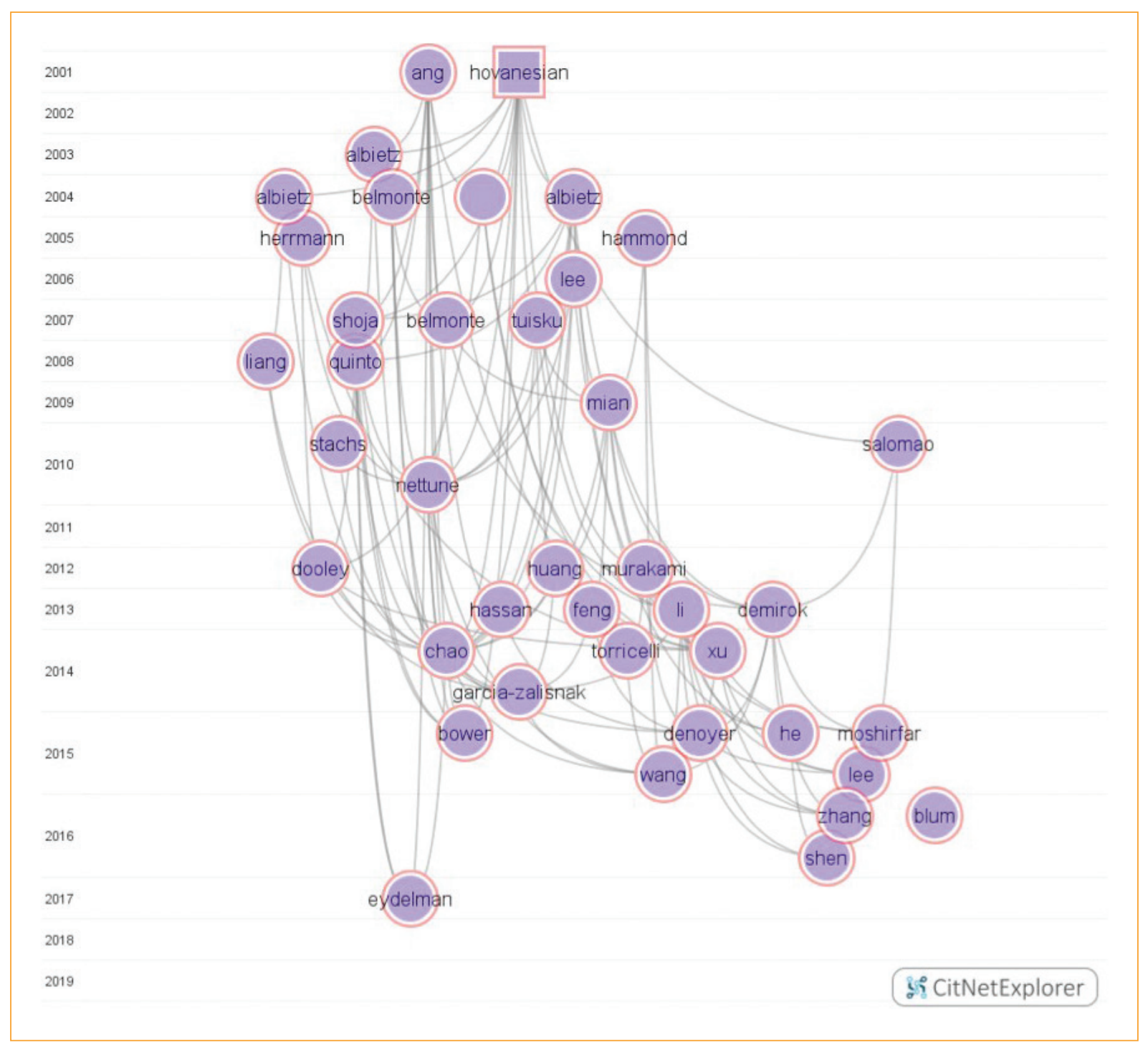

Figura 5. Resultados de la función Core de la red de ojo seco y cirugía refractiva.

sometidos a PRK o LASIK, principalmente por miopía, al menos 6 meses antes. Estos autores concluyeron que los síntomas de ojo seco ocurrieron comúnmente después de LASIK, pero fueron significativamente más graves y prolongados después de PRK. El artículo de Ang, et al. ${ }^{11}$ estudió cómo LASIK y PRK pueden inducir o exacerbar el ojo seco después de la cirugía. Estos autores concluyeron que se debe advertir a los pacientes sobre las complicaciones del ojo seco después de la cirugía y cómo el uso de lágrimas artificiales y tapones de puntos lagrimales son útiles para prevenir 0 aliviar el malestar ocular.

La segunda etapa, de 2002 a 2003, no tiene publicaciones.
La tercera etapa, de 2004 a 2010, incluye los dos años más productivos, 2004 y 2007, con tres publicaciones por año. En 2004 hubo dos publicaciones de Albietz, et al. ${ }^{16,25}$ y una más de Belmonte, et al. ${ }^{20}$ Un artículo de Albietz, et al. ${ }^{16}$ estudió la relación entre el ojo seco crónico y la regresión refractiva después de LASIK, y concluyó que estaba aumentada en los pacientes con ojo seco crónico. El otro artículo de Albietz, et al. ${ }^{25}$ es una revisión de las recomendaciones basadas en la evidencia para las mejores estrategias de manejo en el consultorio de pacientes que sufren ojo seco antes, durante y después de LASIK. Finalmente, el artículo de Belmonte, et al..$^{20}$ estudió la base neural de las sensaciones de la córnea y la conjuntiva después de la cirugía refractiva, y los autores 
concluyeron que la excitabilidad alterada fue el origen de la disminución de la sensibilidad, el dolor espontáneo y la sensación de ojo seco después de la cirugía refractiva. En 2007 encontramos tres artículos: Tuisku, et al..$^{15}$ Shoja, et al. ${ }^{3}$ y Belmonte ${ }^{21}$. Tuisku, et al. ${ }^{15}$ estudiaron a 20 pacientes que se habían sometido a LASIK por miopía alta y compararon los resultados con un grupo control, sin encontrar diferencias demostrables. Shoja, et al. ${ }^{3}$ estudiaron la incidencia y los factores de riesgo del ojo seco después de LASIK por miopía, y concluyeron que estos pacientes desarrollaron ojo seco con función lagrimal comprometida al menos 6 meses después de la cirugía. También encontraron un mayor riesgo de sufrir ojo seco en los pacientes que requirieron una mayor corrección refractiva. El artículo de Belmonte ${ }^{21}$ destacó la inervación de la córnea por tipos funcionales de nervios sensoriales. El autor explica que cuando se estimulan los nervios sensoriales evocan sensaciones como sequedad ocular, malestar y dolor. Concluye que los medicamentos que reducen la actividad anormal en los nervios corneales lesionados podrían representar una alternativa terapéutica para las sensaciones de sequedad ocular después de la cirugía refractiva.

Una etapa de transición ocupa los años 2011 y 2012, sin publicaciones.

La etapa final va de 2013 a 2015, siendo 2015 el año más productivo. Ese año hubo publicaciones con una puntuación de citas de 30, 11 y 11, por Denoyer, et al. ${ }^{9}$, Moshirfar, et al. ${ }^{23}$ y Bower, et al. ${ }^{4}$, respectivamente. El artículo de Denoyer, et al. ${ }^{9}$ estudió la comparación entre la extracción del lentículo por incisión pequeña (SMILE) y LASIK para la enfermedad de ojo seco posrefractiva. Los autores concluyen que SMILE tiene menos impacto en la superficie ocular y la inervación corneal en comparación con LASIK, lo que reduce aún más la incidencia de la enfermedad de ojo seco. El artículo de Moshirfar, et al. ${ }^{23}$ revisó los beneficios, las limitaciones y las aplicaciones futuras del procedimiento SMILE en 56 artículos de la base de datos PubMed. El artículo de Bower, et al. ${ }^{4}$ evaluó las manifestaciones de ojo seco después de PRK y LASIK para determinar la incidencia y los factores predictivos de ojo seco crónico utilizando una serie de criterios. Los autores concluyen que las características de la superficie ocular y la película lagrimal durante el examen preoperatorio podrían ayudar a predecir el desarrollo crónico de ojo seco después de la cirugía. La tercera y quinta etapas destacan la gran relevancia de la investigación en ojo seco y cirugía refractiva en fechas recientes. En resumen, la investigación sobre cirugía refractiva y ojo seco comienza a estudiar los síntomas y signos del ojo seco después de la cirugía, continúa con el estudio de la estructura nerviosa corneal, planteando la hipótesis de que el ojo seco está causado por la amputación de los nervios en el proceso quirúrgico, y finaliza con el estudio de nuevas técnicas que reduzcan la resistencia biomecánica de la córnea, como se pretendía con la técnica SMILE, preservando la membrana de Bowman en el proceso quirúrgico.

En un estudio de red de citación previo sobre el campo del ojo seco, Sánchez-Tena, et al..$^{28}$ encontraron un clúster sobre cirugía refractiva. El artículo más citado en este grupo fue una revisión de Ambrosio, et al. ${ }^{29}$ publicada en 2008 en Journal of Refractive Surgery. Revisaron la literatura científica y resumieron la experiencia de los autores para proponer métodos para disminuir el ojo seco después de la cirugía refractiva. En nuestro estudio de red de citación que se centra en el ojo seco y la cirugía refractiva, ese autor aparece en la posición 3, con una puntuación de citación de 41. La diferencia podría deberse a que el estudio antes mencionado se limitó a publicaciones de 10 años (2007-2018). En nuestro estudio hemos analizado la totalidad de las publicaciones científicas desde el momento de la primera cirugía refractiva.

Gran parte de las referencias seleccionadas se refieren a la técnica LASIK, pero no se hizo distinción en la búsqueda por tipo de cirugía. En el periodo analizado, el mayor número de intervenciones se ha realizado con la técnica LASIK.

Acerca de los procedimientos quirúrgicos más relacionados de los 20 artículos más citados, solo tres de los artículos más citados no hablan sobre LASIK. Esta situación concuerda con la realidad en la clínica, pues LASIK es la técnica más común en todo el mundo. Otra explicación podría ser que LASIK produce una reducción significativa en la producción de lágrimas con respecto a otras técnicas de cirugía refractiva ${ }^{30}$.

Sobre las principales revistas en las que se han publicado los 20 artículos más citados, Journal of Refractive Surgery, Journal of Cataract and Refractive Surgery y Current Opinion in Ophthalmology, estos datos no ofrecen ninguna duda. Tales resultados eran de esperar porque estas revistas son las más importantes en el campo de la cirugía refractiva, y las tres son estadounidenses. Esto podría deberse a una limitación de la base de datos debido a sus preferencias por las revistas en idioma inglés.

En cuanto a los clústeres, se definieron claramente dos grupos. El clúster 1 fue el más grande y la etiología del ojo seco inducido por LASIK fue el tema más 
estudiado. El clúster 2, que era un $81 \%$ más pequeño que el clúster 1 , trataba sobre la cirugía refractiva y otras afecciones relacionadas con el ojo seco. También se hizo referencia al síndrome de ojo seco como punto principal del estudio. Ambos clústeres se diferencian por el enfoque del estudio y los periodos de las publicaciones. La diferencia de tamaño entre ambos grupos puede deberse a la gran cantidad de cirugías LASIK realizadas. Desde que comenzó la técnica LASIK hasta hoy se han realizado decenas de millones de cirugías en todo el mundo, muchas más que intervenciones PRK; por el contrario, la técnica SMILE comenzó a utilizarse en Europa en 2012 y no recibió la aprobación de la Food and Drug Administration hasta 2016. The Carl Zeiss Meditec Company, propietaria del láser de femtosegundo VisuMax para cirugía SMILE, celebró un millón de procedimientos realizados en septiembre de 2017.

Las publicaciones Core representan un alto porcentaje $(47.92 \%)$ en la red. Esto significa que hay un fuerte enfoque en la investigación realizada en este campo. El tratamiento del ojo seco inducido por LASIK está representado en este grupo. Aunque en menor número, también están representadas otras técnicas quirúrgicas y afecciones de ojo seco. La evolución de LASIK desde el uso de un microquerátomo mecánico al de un láser de femtosegundo para crear el colgajo fue para conseguir una mayor predictibilidad de la profundidad del colgajo y una mayor precisión en su espesor, buscando posteriormente menos complicaciones oculares y visuales. Asimismo, las mejoras en PRK, LASEK y Trans-PRK también han proporcionado una mayor previsibilidad y mejores condiciones posoperatorias. En estas publicaciones Core se representan los estudios y las investigaciones que han favorecido estas evoluciones mencionadas. No obstante, el objetivo común ha sido lograr mejoras para el ojo seco en las etapas posteriores a la cirugía. Una forma fue la estrategia de buscar procedimientos que respeten las estructuras más duras de la córnea, como el estroma y la membrana de Bowman, como es el caso de la técnica SMILE. Otra forma fue investigar antes de la cirugía la superficie ocular que podría predecir el ojo seco posquirúrgico.

Las bases de datos bibliográficas son la principal fuente de información utilizada en los estudios bibliométricos. Haber elegido WOS entre otras bases de datos disponibles puede dar lugar a sesgos de información. Las bases de datos del Institute for Scientific Information (ISI) fueron las primeras en permitir el trabajo bibliométrico, y WOS es la única herramienta internacional y multidisciplinaria disponible para acceder a la literatura de varias disciplinas de la ciencia desde hace cuatro décadas. El Journal Citation Report (JCR) se elabora incluyendo las revistas de WOS como citadoras y publicando su factor de impacto. WOS es una herramienta útil tradicional para la búsqueda de bibliografía, aunque también tiene limitaciones; algunas de estas están siendo mejoradas por la base de datos Scopus (Elsevier, 2004), que actualmente indexa más revistas revisadas por pares (SCImago Journal Rank, 2015) que WOS. Si bien una mayor cobertura de Scopus contribuye a la ventaja de contener un mayor número de citas, pierde fuerza para las referencias anteriores a 1996. Esta circunstancia fue de interés para nuestro estudio porque estábamos buscando la literatura más extensa en el tiempo. Otra circunstancia que influyó para que nuestro estudio seleccionara la base de datos de WOS es que enfatiza la calidad de su contenido, más que la cantidad. Esta es una circunstancia que se satisface si recordamos la ley de Bradford, que postula que la mayoría de las publicaciones científicas importantes se encuentran en un número reducido de revistas, aunque esto ha sido criticado por favorecer el idioma inglés americano para la literatura científica. Hasta donde alcanza nuestro conocimiento, no se han realizado estudios para comparar las bases de datos WOS y Scopus en el campo del ojo seco. Consideramos este estudio como una primera parte de comparaciones futuras.

\section{Conclusiones}

El ojo seco tras la cirugía refractiva es un campo muy destacado para los investigadores, con un gran número de publicaciones y conexiones entre ellas. Este estudio aporta conocimientos en cirugía refractiva que servirán para mejorar la aplicabilidad clínica para disminuir el ojo seco.

\section{Conflicto de intereses}

Los autores declaran no tener ningún conflicto de intereses.

\section{Financiamiento}

Esta investigación no recibió financiación externa ni tuvo patrocinadores.

\section{Responsabilidades éticas}

Se trata de un estudio bibliométrico de revisión de la literatura, por lo que no hay intervenciones en pacientes. 
Protección de personas y animales. Los autores declaran que para esta investigación no se han realizado experimentos en seres humanos ni en animales.

Confidencialidad de los datos. Los autores declaran que en este artículo no aparecen datos de pacientes.

Derecho a la privacidad y consentimiento informado. Los autores declaran que en este artículo no aparecen datos de pacientes.

\section{Bibliografía}

1. Craig JP, Nichols KK, Akpek EK, Caffery B, Dua HS, Joo CK, et al. TFOS DEWS II definition and classification report. Ocul Surf. 2017;15:276-83.

2. De Paiva CS, Chen Z, Koch DD, Hamill MB, Manuel FK, Hassan SS, et al. The incidence and risk factors for developing dry eye after myopic LASIK. Am J Ophthalmol. 2006;141:438-45.

3. Shoja MR, Besharati MR. Dry eye after LASIK for myopia: incidence and risk factors. Eur J Ophthalmol. 2007;17:1-6.

4. Bower KS, Sia RK, Ryan DS, Mines MJ, Dartt DA. Chronic dry eye in photorefractive keratectomy and laser in situ keratomileusis: manifestations, incidence, and predictive factors. J Cataract Refract Surg. 2015;41:2624-34

5. CitNetExplorer@: Analyzing citation patterns in scientific literature. [Sitio Web de Citation Network Explorer]. 2019. Disponible en: http://www.citnetexplorer.nl. Consultado el 8 de febrero de 2019.

6. Van Eck NJ, Waltman L. CitNetExplorer: una nueva herramienta de software para analizar y visualizar la red de citación. Journal of Informetrics. 2014;8(4)

7. Van Eck NJ, Waltman L. Citation-based clustering of publications using CitNetExplorer and VOSviewer. Scientometrics. 2017:111:1053-70.

8. Hovanesian JA, Shah SS, Maloney RK. Symptoms of dry eye and recurrent erosion síndrome after refractive surgery. J Cataract Refract Surg 2001;27:577-84.

9. Denoyer A, Landman E, Trinh L, Faure JF, Auclin F, Baudouin C. Dry eye disease after refractive surgery: comparative outcomes of small in cision lenticule extraction versus LASIK. Ophthalmology. 2015;122 669-76.

10. Li M, Zhao J, Shen Y, Li T, Xu H, Yu Y, et al. Comparison of dry eye and corneal sensitivity between small incision lenticule extraction and femtosecond LASIK for myopia. Plos One. 2013;8:e77797.

11. Ang RT, Dartt DA, Tsubota K. Dry eye after refractive surgery. Curr Opin Ophthalmol. 2001;12:318-22.
12. Xu Y, Yang $Y$. Dry eye after small incision lenticule extraction and LASIK for myopia. J Refract Surg. 2014;30:186-90.

13. Demirok A, Ozgurhan EB, Agca A, Kara N, Bozkurt E, Cancaya KI, et al. Corneal sensation after corneal refractive surgery with small incision lenticule extraction. Optom Vis Sci. 2013;90:1040-7.

14. Nettune GR, Pflugfelder SC. Post-LASIK tear dysfunction and dysesthesia. The Ocul Surf. 2010:8:135-45.

15. Tuisku IS, Lindbohm N, Wilson SE, Tervo TM. Dry eye and corneal sensitivity after high myopic LASIK. J Refract Surg. 2007;23:338-42.

16. Albietz JM, Lenton LM, McLennan SG. Chronic dry eye and regression after laser in situ keratomileusis for myopia. J Cataract Refract Surg. 2004;30:675-84

17. Mian S, Li AY, Dutta S, Musch DC, Shtein RM. Dry eyes and corneal sensation after laser in situ keratomileusis with femtosecond laser flap creation. Effect of hinge position, hinge angle, and flap thickness. $\mathrm{J}$ Cataract Refract Surg. 2009;35:2092-8.

18. Chao C, Golebiowski B, Stapleton $F$. The role of corneal innervation in LASIK-induced neuropathic dry eye. Ocul Surf. 2014;12:32-45.

19. Quinto GG, Camacho W, Behrens A. Postrefractive surgery dry eye. Curr Opin Ophthalmol. 2008;19:335-41.

20. Belmonte C, Acosta MC, Gallar J. Neural basis of sensation in intact and injured corneas. Exp Eye Res. 2004;78:513-25.

21. Belmonte, C. Eye dryness sensations after refractive surgery: impaired tear secretion or "phantom" cornea? J Refract Surg. 2007;23:598-602.

22. Zhang $Y$, Shen Q, Jia Y, Zhou D, Zhou J. Clinical outcomes of SMILE and FS-LASIK used to treat myopia: a meta-analysis. J Refract Surg. 2016:32:256-65.

23. Moshirfar M, McCaughey MV, Dan BS, Reinstein Z, Shah R, Santiago-Caban L. Small-incision lenticule extraction. J Cataract Refract Surg. 2015;41:652-65.

24. Lee SJ, Kim JK, Seo KY, Kim EK, Lee HK. Comparison of corneal nerve regeneration and sensitivity between keratomileusis (LASEK). Am J Ophthalmol. 2006;141:1009-15.

25. Albietz JM, Lenton LM. Management of the ocular surface and tear film before, during, and after laser in situ keratomileusis. J Refract Surg. 2004;20:62-71.

26. Liang L, Zhang M, Zou W, Liu Z. Aggravated dry eye after laser in situ keratomileusis in patients with Sjögren syndrome. Cornea. 2008;27:120-3.

27. Lemp MA. Report of the National Eye Institute/Industry workshop on Clinical Trials in Dry Eyes. CLAO J. 1995;21:221-32.

28. Sánchez-Tena MA, Álvarez-Peregrina C, Villa-Collar C. Dry eye analysis: a citation network study. J Ophthalmol. 2019;2019:3048740.

29. Ambrosio R, Tervo T, Wilson SE. LASIK-associated dry eye and neurotrophic epitheliopathy: pathophysiology and strategies for prevention and treatment. J Refract Surg. 2008;24:396-407.

30. Sambhi RS, Shambh GDS, Mather R, Malvankar-Mehta MS. Dry eye after refractive surgery: a meta-analysis. Can J Ophthalmol. 2020;55:99-106. 